\title{
Combined interventional and surgical treatment of tandem middle cerebral artery embolus and internal carotid artery occlusion: case report
}

\author{
Mark B. Frenkel, MD, Jaclyn J. Renfrow, MD, Jasmeet Singh, MD, ${ }^{2}$ Nitin Garg, MD, ${ }^{3}$ and \\ Stacey Q. Wolfe, MD'
} Departments of ${ }^{1}$ Neurological Surgery, ${ }^{2}$ Radiology, and ${ }^{3}$ Vascular and Endovascular Surgery, Wake Forest School of Medicine,
Winston-Salem, North Carolina

\begin{abstract}
Tandem internal carotid artery (ICA) origin occlusion and middle cerebral artery (MCA) thromboembolism is a life-threatening condition with poor neurological outcome. The authors report on a patient presenting with acute ischemic stroke from a tandem ICA and MCA occlusion with penumbra. Emergency MCA mechanical thrombectomy was performed through percutaneous cervical ICA access due to the inability to cross the cervical carotid occlusion. Emergency carotid endarterectomy to reperfuse the poorly collateralized hemisphere and repair the ICA access site was performed 2 hours after completion of tissue plasminogen activator (tPA) infusion. This case illustrates the shortest reported interval between IPA infusion and open surgical intervention for carotid revascularization, as well as the role of direct carotid artery access for mechanical thrombectomy. The authors also describe the use of a temporizing femoral artery-to-ICA shunt to maintain cerebral perfusion in the setting of ICA occlusion.
\end{abstract}

https://thejns.org/doi/abs/10.3171/2017.6.JNS162368

KEY WORDS stroke; tPA; tandem occlusion; carotid endarterectomy; intracranial thrombectomy; vascular disorders

A CUTE extracranial internal carotid artery (eICA) occlusion with tandem distal occlusion represents a clinical challenge. Ischemic strokes in patients with eICA occlusions are much more severe and have worse outcomes than with ICA stenosis alone, ${ }^{1}$ and tandem ICA/middle cerebral artery (MCA) occlusion is an independent predictor of poor recanalization and poor clinical outcome after intravenous thrombolysis. ${ }^{22}$ Given the potentially devastating outcome of tandem occlusions, intervention is indicated. Simultaneous ICA stenting and intracranial thrombectomy has been described with good results. ${ }^{14,24}$ However, this approach requires successful ICA recanalization for distal access and reperfusion.

We report a case of carotid endarterectomy (CEA) immediately following intracranial thrombectomy through percutaneous cervical ICA access due to failed attempts to traverse a bulky atherosclerotic plaque at the ICA origin. This case illustrates the shortest reported interval between intravenous tissue plasminogen activator (tPA) infusion and surgical intervention, the potential role of acute surgical carotid revascularization, and the role of direct carotid artery puncture for difficult carotid access. We also describe the creation of a temporizing shunt from a femoral artery-to-ICA sheath that was used preoperatively to maintain cerebral perfusion and prevent distal reocclusion in the absence of antegrade ICA flow and poor collateral blood supply.

\section{Case Report}

History and Examination

A 71-year-old man with hyperlipidemia, hypertension, and an anaphylactic allergy to aspirin had a witnessed collapse in the hospital cafeteria. He demonstrated left hemiplegia and neglect with a National Institutes of Health Stroke Scale (NIHSS) score of 18. A CT scan of his head was normal (Alberta stroke program early CT score [ASPECTS] of 10) and a CT arteriogram demonstrated a right eICA occlusion at the origin with a heavily calcified atheromatous plaque (Fig. 1A and B). Supraclinoid ICA

ABBREVIATIONS CCA = common carotid artery; $C E A=$ carotid endarterectomy; elCA = extracranial ICA; ICA = internal carotid artery; MCA = middle cerebral artery; NIHSS = National Institutes of Health Stroke Scale; $\mathrm{TICl}=$ thrombolysis in cerebral infarction; tPA = tissue plasminogen activator.

SUBMITTED January 19, 2017. ACCEPTED June 2, 2017.

INCLUDE WHEN CITING Published online November 17, 2017; DOI: 10.3171/2017.6.JNS162368. 

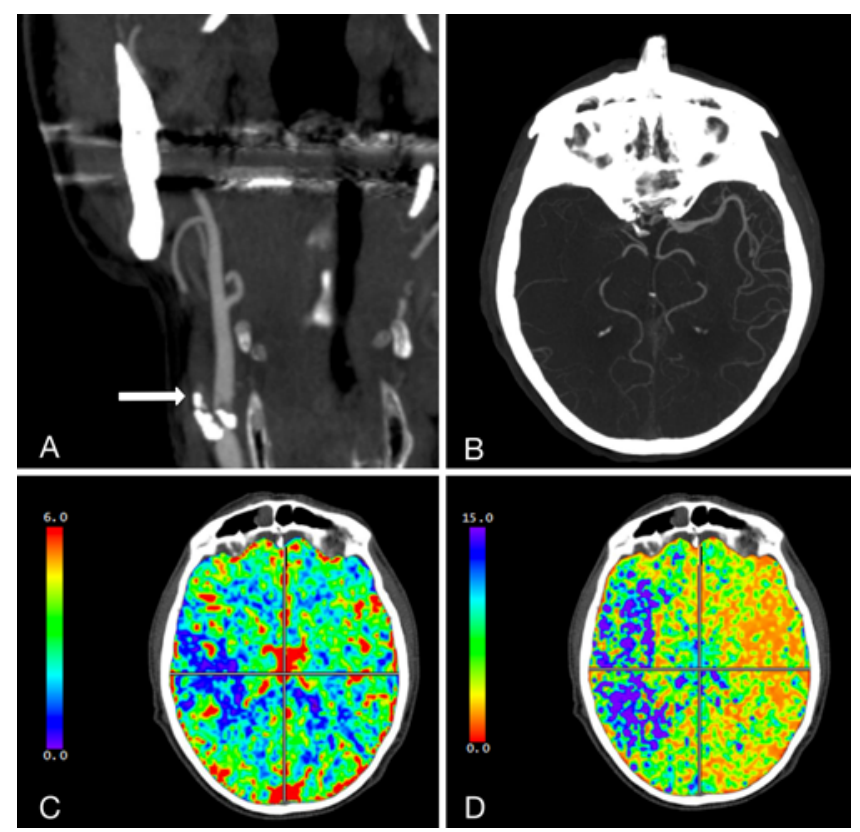

FIG. 1. A: Coronal CT arteriogram image demonstrating a right-sided ICA occlusion at the origin (arrow) with a heavily calcified atheromatous plaque. B: Axial CT arteriogram image demonstrating right-sided $M_{1}$ occlusion. C and D: CT perfusion blood volume $(C)$ and mean transit time (D) images demonstrating a central patchy MCA with a large penumbra involving the entire MCA territory.

reconstitution was noted with a tandem right $\mathrm{M}_{1}$ occlusion and poor collateral flow. CT perfusion showed a central patchy MCA infarct (less than one-third the MCA territory) on blood volume imaging with a large penumbra involving the entire MCA territory (Fig. 1C and D).

\section{Interventions}

The patient received a $0.09 \mathrm{mg} / \mathrm{kg}$ bolus of intravenous tPA (alteplase) within 20 minutes of symptom onset, followed by a $0.81 \mathrm{mg} / \mathrm{kg}$ infusion over the next hour. He was taken to the neurointerventional suite for revascularization within 30 minutes of symptom onset. The right femoral artery was accessed. Selective angiography of the right common carotid artery (CCA) showed near-occlusion of the right ICA origin with stasis at the carotid bulb and absence of distal flow (Fig. 2A). Multiple attempts were made to traverse the proximal ICA lesion without success using a variety of microcatheters, wires, and techniques, including a 0.035 -inch hydrophilic guidewire and a Gateway $1.5 \times 9 \mathrm{~mm}$ balloon (Boston Scientific) over a Synchro 2 guidewire (Stryker Neurovascular). Given the short time from onset of symptoms, salvageable penumbra, lack of clinical improvement with tPA, and magnitude of the patient's deficits, we elected to percutaneously access the cervical eICA distal to the occlusion to perform MCA thrombectomy. After ultrasound-guided micropuncture access, a 6-Fr $\times 10 \mathrm{~cm}$ sheath was placed with no backflow. A 6-Fr guide catheter (Envoy DA XB, Codman Neuro) was placed into the distal cervical ICA and weak retrograde ICA flow was noted in the sheath after suction aspiration thrombectomy through the guide catheter. Follow-up angiography demonstrated a patent supraclinoid ICA but right $\mathrm{M}_{1}$ occlusion (Fig. 2B). Thrombectomy was performed with a single pass of the Solitaire $(4 \times 40 \mathrm{~mm})$ clot retrieval system (Medtronic). Complete revascularization (thrombolysis in cerebral infarction [TICI] Grade 3) was achieved 101 minutes from the time of femoral access, 15 minutes from carotid access, and just over 2 hours from symptom onset (Fig. 2C). Despite a patent anterior communicating artery, sluggish retrograde flow was noted in the carotid sheath after completion of thrombectomy, consistent with insufficient collateral perfusion. Reexamination showed the patient to be following commands in his left lower extremity with $3 / 5$ strength, although the upper extremity was still plegic.

The patient was taken to the operating room emergently for CEA to restore adequate antegrade flow and for removal of the carotid sheath under direct visualization. To maintain perfusion of the poorly collateralized distal ICA territory during transport, low-dose heparin bolus (40 units/kg, given the presence of acute cerebral infarction) was given intravenously, and the femoral artery and ICA sheaths were connected via intravenous tubing and a

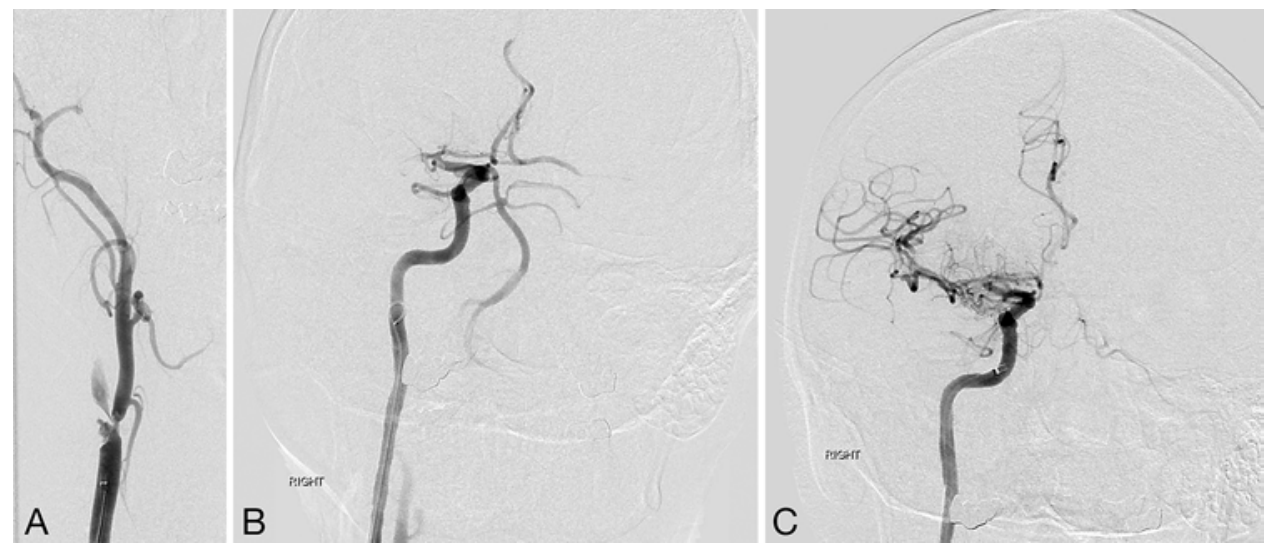

FIG. 2. Digital subtraction angiography anteroposterior images demonstrating initial near-occlusion of the right ICA origin with stasis at the carotid bulb and absence of distal flow $(A)$, right $\mathrm{M}_{1}$ occlusion after percutaneous ICA access (B), and postthrombectomy $\mathrm{TICl}$ Grade 3 right MCA reperfusion (C). 


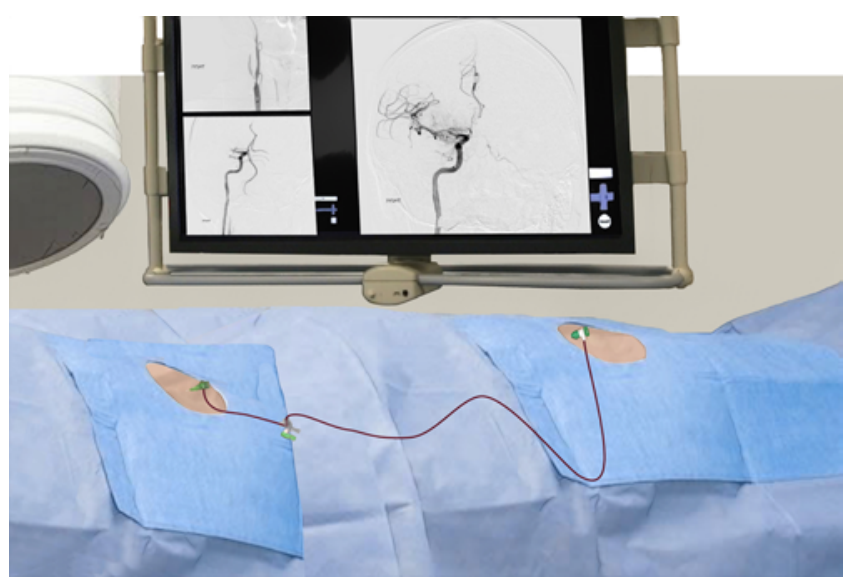

FIG. 3. Artist's rendition of extracorporeal shunt between femoral artery and ICA sheaths allowing for antegrade ICA perfusion during patient transport. Copyright Mark Frenkel. Published with permission.

male-to-male connector (Fig. 3). An arterial line sensor was attached to the circuit to monitor flow. An oblique incision lateral to the carotid sheath was made 3 hours from symptom onset under general anesthesia. After dissection and clamping of the ICA with standard technique, the arteriotomy was made to incorporate the catheter site, and the sheath was removed. CEA was performed with shunt placement and patch angioplasty in a standard fashion. Excellent hemostasis was observed and placement of a surgical drain was believed to be unnecessary. The procedure was technically uncomplicated with no significant or abnormal bleeding encountered. The procedure lasted 2 hours, and concluded approximately 4 hours after the completion of the tPA infusion and 5 hours from symptom onset.

\section{Postoperative Course}

Postoperative MRI showed scattered subcortical areas of restricted diffusion in the right MCA territory without hemorrhagic conversion (Fig. 4). Given the patient's severe aspirin allergy, he was started on a heparin drip without bolus with a partial thromboplastin time goal of 50-70 seconds to reduce the risk of thromboembolic events following CEA. He was transitioned from heparin to clopidogrel on the 1st postoperative day. At that time, he demonstrated $4 / 5$ strength in his left leg and $2 / 5$ strength in his left arm, which improved to $4 / 5$ strength on the 2 nd postoperative day. He was discharged to inpatient rehabilitation on postoperative Day 7. At the 6-month follow-up evaluation, the patient had 4/5 strength in his left upper extremity, was able to ambulate with minimal assistance, and had no swallowing or cognitive difficulties (modified Rankin Scale [mRS] score of 2). The patient returned to work after 9 months. At 1 year, he still has decreased range of motion in his left shoulder from adhesive capsulitis and mild left-sided neglect (mRS score of 1).

\section{Discussion}

Patients who suffer from tandem occlusions not only have worse clinical outcomes and higher mortality rates

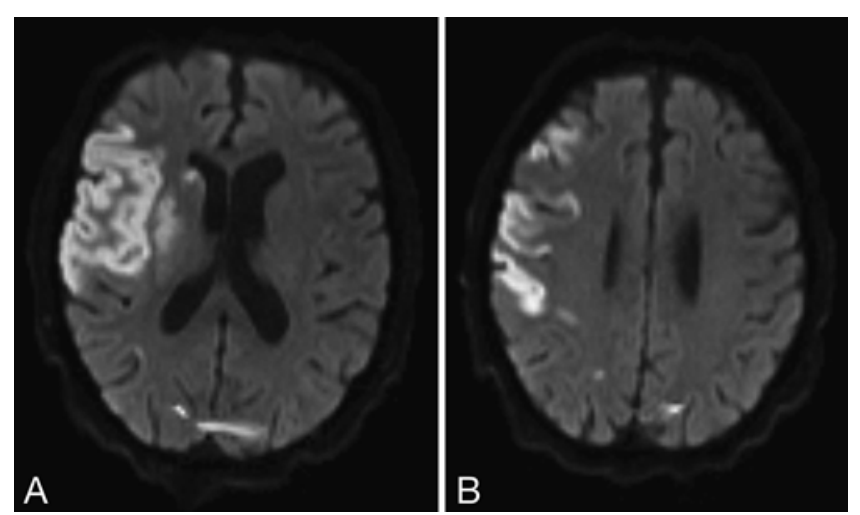

FIG. 4. Axial diffusion-weighted MR images obtained on postoperative Day 1 demonstrating scattered cortical and subcortical areas of restricted diffusion in the right MCA territory, without hemorrhagic conversion. Note that the region of high signal intensity in the bilateral occipital lobes is artifactual.

compared with patients with isolated MCA occlusions, but are less likely to respond to intravenous tPA.22 Awareness of this poor response to medical therapy has led to increased use of interventional thrombectomy, with revascularization rates ranging from $73 \%$ to $100 \%$ and clinical outcomes significantly better than the natural history of this condition. ${ }^{7,16,18,20,24} \mathrm{CEA}$ in the modern era for acute stroke from tandem lesions after thrombectomy has rarely been described in the literature. One group reported thrombolytic therapy and intracranial thrombectomy followed by delayed CEA for a partially occlusive eICA thrombus,$^{13}$ and another group reported on 3 patients who underwent emergency surgical embolectomy of ICA terminus emboli followed by CEA with significant postoperative improvements in their NIHSS scores (median 17 points)..$^{12}$

Historically, CEA for ICA occlusion in acute stroke was associated with an increased risk of intracranial hemorrhage and poor clinical outcome, ${ }^{6}$ and these concerns are amplified in patients who receive thrombolytics. However, in the modern era of endovascular therapy and penumbra imaging, this may be a reasonable option in selected patients. A recent series of 22 patients undergoing emergency CEA for carotid occlusion showed a $72 \%$ revascularization rate with a $77 \%$ improvement in NIHSS score ( $\geq 2$ points within 24 hours of surgery). Nine patients had tandem intracranial lesions, and all underwent surgery within 450 minutes of symptom onset. Five patients received intravenous tPA prior to surgery with 1 postoperative intracranial hemorrhage. ${ }^{4}$ Another case report of emergency CEA after intravenous tPA for acute eICA occlusions without tandem lesions also showed favorable outcomes. ${ }^{9}$ While intravenous tPA has been reported as a risk factor for intracerebral hemorrhage after CEA, ${ }^{26}$ other studies suggest it is not a significant concern in properly selected patients. ${ }^{2,3,19}$ These modern findings of CEA for acute occlusion, even in the setting of thrombolytic administration, mirror the endovascular experience of acute carotid stenting for acute occlusion and stroke, which demonstrates high revascularization rates, good clinical outcomes, and an acceptable safety profile..$^{10,17,25}$ Further- 
more, we believe that in appropriate circumstances, surgery may remain a viable option after tPA infusion given its short half-life ( $<5$ minutes).

Challenging carotid anatomy is associated with worse clinical outcome in acute stroke, due to significant time delay for catheter access and lower rates of recanalization. ${ }^{21}$ While peripheral access is now the preferred route, direct common carotid artery (CCA) puncture has been described to obtain access in difficult anatomy, such as tortuous or atherosclerotic vessels,, 523 similar to the original method of cerebral angiography as developed by Egas Moniz in 1927. ${ }^{27}$ A recent forum discussion among neurointerventionalists highlighted the potential use of cervical ICA access with ultrasound guidance if transfemoral access could not be secured within 15 minutes, although concerns were raised as ICA access, necessary for treatment of intracranial thromboembolism, is more challenging than CCA access (www.snisonline.org, accessed on July 28, 2016).

Closure of an ICA arteriotomy from direct puncture is also challenging, as it is a noncompressible area, especially in the face of thrombolytic, antiplatelet, and anticoagulation medications. The principles of single wall puncture, heparin reversal prior to sheath removal, and close airway monitoring are paramount. ${ }^{5}$ Off-label use of Angio-Seal (St. Jude Medical Inc.) has been reported in CCA punctures with good outcomes; ${ }^{8}$ however, the ICA is often smaller than that indicated for a closure device (4 $\mathrm{mm}$ or larger vessel).

Intracranial thrombectomy is ultimately only successful if there is perfusion of the affected territory, and every 30 minutes without adequate perfusion results in worsened clinical outcome. ${ }^{15}$ Use of a side port shunt for antegrade lower extremity perfusion with large sheath access is well described in patients requiring the endovascular repair of complex aneurysms and extracorporeal membrane oxygenation. ${ }^{11}$ We adapted this technique to harness the pressure gradient of normal peripheral arterial flow to perfuse the poorly collateralized occluded carotid territory. It must be noted that this is a novel strategy used in a unique situation with associated risk given the need for heparinization to prevent thrombosis of the shunt tubing. We balanced the potential risk of intracranial hemorrhage from low-dose heparinization in an acute stroke with the risk of ongoing ischemia and tissue loss, and monitored for thrombosis of the shunt tubing with an arterial waveform monitor. While successful in this case, more data are required prior to concluding that this is safe and/or efficacious.

We describe a case of acute tandem ICA and MCA occlusion that failed to respond to thrombolytic therapy. Given the poor prognosis of this condition, our multidisciplinary stroke team used a unique combination of techniques to achieve revascularization. Thrombectomy via percutaneous ICA puncture followed by immediate CEA approximately 2 hours after completion of the tPA dose makes this the earliest published report of CEA performed after intravenous tPA. While there is no way to fully ascertain the impact of our treatment approach, this patient's near-complete recovery despite a poor presentation supports the possible utility of emergency revascu- larization through combined endovascular and surgical means, regardless of recent intravenous thrombolytic administration.

\section{References}

1. Adams HP Jr, Bendixen BH, Leira E, Chang KC, Davis PH, Woolson RF, et al: Antithrombotic treatment of ischemic stroke among patients with occlusion or severe stenosis of the internal carotid artery: A report of the Trial of Org 10172 in Acute Stroke Treatment (TOAST). Neurology 53:122-125, 1999

2. Azzini C, Gentile M, De Vito A, Traina L, Sette E, Fainardi E, et al: Very early carotid endarterectomy after intravenous thrombolysis. Eur J Vasc Endovasc Surg 51:482-486, 2016

3. Bazan HA, Zea N, Jennings B, Smith TA, Vidal G, Sternbergh WC III: Urgent carotid intervention is safe after thrombolysis for minor to moderate acute ischemic stroke. J Vasc Surg 62:1529-1538, 2015

4. Beneš V, Buchvald P, Klimošová S, Eichlová Z, Suchomel P: Acute extracranial occlusion of the internal carotid artery: emergent surgery remains a viable option. Acta Neurochir (Wien) 156:901-909, 2014

5. Blanc R, Piotin M, Mounayer C, Spelle L, Moret J: Direct cervical arterial access for intracranial endovascular treatment. Neuroradiology 48:925-929, 2006

6. Bruetman ME, Fields WS, Crawford ES, Debakey ME: Cerebral hemorrhage in carotid artery surgery. Arch Neurol 9:458-467, 1963

7. Cohen JE, Leker RR, Eichel R, Gomori M, Itshayek E: Emergency endovascular revascularization of tandem occlusions: Internal carotid artery dissection and intracranial large artery embolism. J Clin Neurosci 28:157-161, 2016

8. Cuellar H, Guimaraens L, Ambekar S, Vivas E, Theron J: Angioseal $^{\mathrm{TM}}$ as a hemostatic device for direct carotid puncture during endovascular procedures. Interv Neuroradiol 21:273-276, 2015

9. Curtze S, Putaala J, Saarela M, Vikatmaa P, Kantonen I, Tatlisumak T: Carotid embolectomy and endarterectomy for symptomatic complete occlusion of the carotid artery as a rescue therapy in acute ischemic stroke. Case Rep Neurol 3:301-308, 2011

10. Du Mesnil De Rochemont R, Sitzer M, Neumann-Haefelin T, Harmjanz A, Berkefeld J: Endovascular recanalization of acute atherothrombotic carotid artery occlusion holds up progressive stroke. Neuroradiology 46:583-586, 2004

11. Hanley SC, Neequaye SK, Steinmetz O, Obrand D, Mackenzie K, Abraham CZ: Sheath-shunt technique for avoiding lower limb ischemia during complex endovascular aneurysm repair. J Vasc Surg 62:762-766, 2015

12. Hasegawa H, Inoue T, Tamura A, Saito I: Emergent intracranial surgical embolectomy in conjunction with carotid endarterectomy for acute internal carotid artery terminus embolic occlusion and tandem occlusion of the cervical carotid artery due to plaque rupture. J Neurosurg 122:939-947, 2015

13. Hinman JD, Rao NM, Yallapragada A, Capri J, Souda P, Whitelegge J, et al: Drip, ship, and grip, then slice and dice: comprehensive stroke center management of cervical and intracranial emboli. Front Neurol 4:104, 2013

14. Jovin TG, Gupta R, Uchino K, Jungreis CA, Wechsler LR, Hammer MD, et al: Emergent stenting of extracranial internal carotid artery occlusion in acute stroke has a high revascularization rate. Stroke 36:2426-2430, 2005

15. Khatri P, Abruzzo T, Yeatts SD, Nichols C, Broderick JP, Tomsick TA: Good clinical outcome after ischemic stroke with successful revascularization is time-dependent. Neurology 73:1066-1072, 2009

16. Lescher S, Czeppan K, Porto L, Singer OC, Berkefeld J: Acute stroke and obstruction of the extracranial carotid ar- 
tery combined with intracranial tandem occlusion: results of interventional revascularization. Cardiovasc Intervent Radiol 38:304-313, 2015

17. Levy DI: Endovascular treatment of carotid artery occlusion in progressive stroke syndromes: technical note. Neurosurgery 42:186-193, 1998

18. Mbabuike N, Gassie K, Brown B, Miller DA, Tawk RG: Revascularization of tandem occlusions in acute ischemic stroke: review of the literature and illustrative case. Neurosurg Focus 42(4):E15, 2017

19. McPherson CM, Woo D, Cohen PL, Pancioli AM, Kissela BM, Carrozzella JA, et al: Early carotid endarterectomy for critical carotid artery stenosis after thrombolysis therapy in acute ischemic stroke in the middle cerebral artery. Stroke 32:2075-2080, 2001

20. Rangel-Castilla L, Rajah GB, Shakir HJ, Shallwani H, Gandhi S, Davies JM, et al: Management of acute ischemic stroke due to tandem occlusion: should endovascular recanalization of the extracranial or intracranial occlusive lesion be done first? Neurosurg Focus 42(4):E16, 2017

21. Ribo M, Flores A, Rubiera M, Pagola J, Mendonca N, Rodriguez-Luna D, et al: Difficult catheter access to the occluded vessel during endovascular treatment of acute ischemic stroke is associated with worse clinical outcome. J Neurointerv Surg 5 (Suppl 1):i70-i73, 2013

22. Rubiera M, Ribo M, Delgado-Mederos R, Santamarina E, Delgado P, Montaner J, et al: Tandem internal carotid artery/ middle cerebral artery occlusion: an independent predictor of poor outcome after systemic thrombolysis. Stroke 37:23012305, 2006

23. Samaniego EA, Dabus G, Raju R, Tsoukas AI, Linfante I: Intracranial angioplasty and stenting through direct carotid puncture. J Neuroimaging 23:207-210, 2013

24. Stampfl S, Ringleb PA, Möhlenbruch M, Hametner C, Herweh C, Pham M, et al: Emergency cervical internal carotid artery stenting in combination with intracranial thrombec- tomy in acute stroke. AJNR Am J Neuroradiol 35:741-746, 2014

25. Tsivgoulis G, Safouris A, Katsanos AH, Arthur AS, Alexandrov AV: Mechanical thrombectomy for emergent large vessel occlusion: a critical appraisal of recent randomized controlled clinical trials. Brain Behav 6:e00418, 2016

26. Vellimana AK, Yarbrough CK, Blackburn S, Strom RG, Pilgram TK, Lee JM, et al: Intravenous tissue-type plasminogen activator therapy is an independent risk factor for symptomatic intracerebral hemorrhage after carotid endarterectomy. Neurosurgery 74:254-261, 2014

27. Wilkins RH, Moniz E: Neurosurgical Classic-XVI. Arterial encephalography, its importance in the localization of cerebral tumors. J Neurosurg 21:144-156, 1964

\section{Disclosures}

The authors report no conflict of interest concerning the materials or methods used in this study or the findings specified in this paper.

\section{Author Contributions}

Conception and design: Wolfe, Frenkel. Acquisition of data: Wolfe, Frenkel, Singh, Garg. Analysis and interpretation of data: Wolfe, Frenkel, Renfrow, Singh. Drafting the article: Frenkel, Renfrow. Critically revising the article: Wolfe, Frenkel, Renfrow, Garg. Reviewed submitted version of manuscript: Wolfe, Frenkel, Singh, Garg. Approved the final version of the manuscript on behalf of all authors: Wolfe. Study supervision: Wolfe, Frenkel.

\section{Correspondence}

Stacey Q. Wolfe, Department of Neurological Surgery, Wake Forest School of Medicine, 1 Medical Center Blvd., Winston-Salem, NC 27157. email: sqwolfe@wakehealth.edu. 